\title{
Retropharyngeal emphysema: An unusual aetiology-A case report and literature review ${ }^{*}$
}

\author{
Daniel Adekunle, Onakoya Paul Adekunle \\ Department of Otorhinolaryngology, College of Medicine and University College Hospital, Ibadan, Nigeria; \\ ${ }^{\#}$ Corresponding Authors: kunle_d2002@yahoo.com, paonak@gmail.com
}

Received 12 March 2013; revised 15 April 2013; accepted 1 May 2013

Copyright (C 2013 Daniel Adekunle, Onakoya Paul Adekunle. This is an open access article distributed under the Creative Commons Attribution License, which permits unrestricted use, distribution, and reproduction in any medium, provided the original work is properly cited.

\section{ABSTRACT}

Introduction: Retropharyngeal emphysema is a potentially life threatening condition which may require urgent diagnosis and intervention. It has been documented to occur following trauma to the aerodigestive tract and is usually accompanied by subcutaneous emphysema of the cervical region. This condition may be complicated by mediastinal emphysema and pneumomediastinum. Foreign body ingestion is quite common in little children and in this environment; a cultural practice is for mothers or other caregivers to attempt to remove these foreign bodies by forcing their finger(s) down the oropharynx and Hypopharynx of these young children. This usually results in injuries to these anatomical sites. Retropharyngeal emphysema complicating this practice is rarely reported. Case presentation: We present the case of a 4-year-old Nigerian boy who presented with stridor and severe dyspnoea following repeated attempts at removal of a purportedly ingested foreign body by his mother. Examination revealed oedema of the soft palate with small multiple bleeding shallow lacerations of the oropharyngeal walls. Oxygen therapy was commenced and preparations for urgent upper airway intervention made. Soft tissue neck X-ray revealed retropharyngeal and subcutaneous emphysema with upper airway compromise. Conservative treatment with high dose steroids and antibiotics led to a gra-

\footnotetext{
*Competing interest: The authors declare that they have no competing interests.

Author's Contributions: DA was the principal investigator, performed literature search and wrote the manuscript. OPA assisted in preparing and proofreading the manuscript for intellectual content and gave final approval for the publication. DA and OPA take responsibility for the publication of the manuscript.
}

dual resolution of the condition. Conclusion: This is a rarely reported complication of this relatively common socio-cultural "foreign body removal method". Public awareness is required to stop this potentially life threatening practice.

Keywords: Retropharyngeal Emphysema; Foreign Body; Foreign Body Removal

\section{INTRODUCTION}

Retropharyngeal emphysema is a severe condition which may be life threatening requiring urgent diagnosis and intervention. This condition may occur with subcutaneous emphysema of the cervical region and may also be complicated by mediastinal emphysema and pneumomediastinum [1].

Foreign body ingestion is very common amongst the paediatric age group, especially in our environment [2]. When these foreign bodies are ingested, the mothers or other caregivers usually would have made several attempts at removal. The resultant effect is usually injury to the mucosa and soft tissues of the oral cavity and oropharynx at presentation. Upper airway compromise is very rare in these patients. Whenever upper airway compromise is observed in some cases, it is often due to laryngeal oedema following trauma.

This potentially fatal practice is still rife and hence we present the case of a 4-year-old boy whose mother attempted to remove a foreign body from his throat with resultant life threatening retropharyngeal and subcutaneous emphysema; and a review of literature.

\section{CASE REPORT}

A 4-year-old Nigerian boy was brought into the children's emergency room with a 5-hour history of progressively worsening dyspnoea. There was history of possi- 
ble foreign body ingestion, though with no eye witness account and no symptoms attributable to foreign body in the aerodigestive tract. His mother assumed he had ingested a cosmetic bead (which he erstwhile had been playing with) when she could no longer find it.

She made several attempts at removal by thrusting the index and middle fingers of her right hand down his throat while holding him in a head down position. This procedure was discontinued when blood was observed in the child's mouth; followed later by progresssive dyspnoea. There was neither hoarseness nor haematemesis nor cough but he developed a low grade fever. There was also a progressive painless swelling of the lower aspect of the neck. Systemic review was essentially normal. There was no intercurrent medical condition.

Examination revealed a severely dyspnoeic child with suprasternal, intercoastal and sub-costal recessions. His respiratory rate was 48 cycles per minute. There was mild inspiratory stridor. His temperature was 38.3 degrees centigrade and pulse oximetry revealed oxygen saturation ranging from $89 \%$ to $93 \%$ in room air. Chest percussion notes were resonant and auscultation revealed mild inspiratory and expiratory ronchi in both right and left lung fields. We did not have facilities for arterial blood gases.

His oropharynx revealed bleeding from multiple fairly deep lacerations in the tonsillar pillars, uvula and posterior pharyngeal wall. The posterior pharyngeal wall was bulging. Examination of the neck revealed minimal painless swelling in both supraclavicular fossae with crepitus.

Urgent Roentgenograms of the neck and chest were then requested. The lateral X-ray of the soft tissue neck revealed widening of and lucency within the prevertebral soft tissue from the basiocciput, extending below the $7^{\text {th }}$ cervical vertebra; most marked at the level of the $1^{\text {st }}$ to $3^{\text {rd }}$ cervical vertebrae, which was suggestive of retropharyngeal emphysema. This has narrowed supraglottic region with the posterior pharyngeal wall abutting on the tip of the epiglottis. No foreign body was seen in the laryngeal or tracheal air column. Streaks of air were also seen in the subcutaneous tissue of the neck (Figure 1). The chest X-ray did not reveal any evidence of mediastinal emphysema or pneumo-mediastinum (Figure 2).

He was nursed in a semi recumbent position and supplemental humidified oxygen at $100 \%$ was administered via a facemask. There was immediate improvement in the oxygen saturation (ranged from 97\% to 100\%). High dose intravenous steroids (Hydrocortisone $80 \mathrm{mg}$ every 8 hours for 48 hours) and broad spectrum antibiotics (Cefuroxime at $500 \mathrm{mg}$ every 8 hours and Metronidazole at $120 \mathrm{mg}$ every 8 hours for 48 hours) were commenced and close monitoring of the patient with quarter hourly

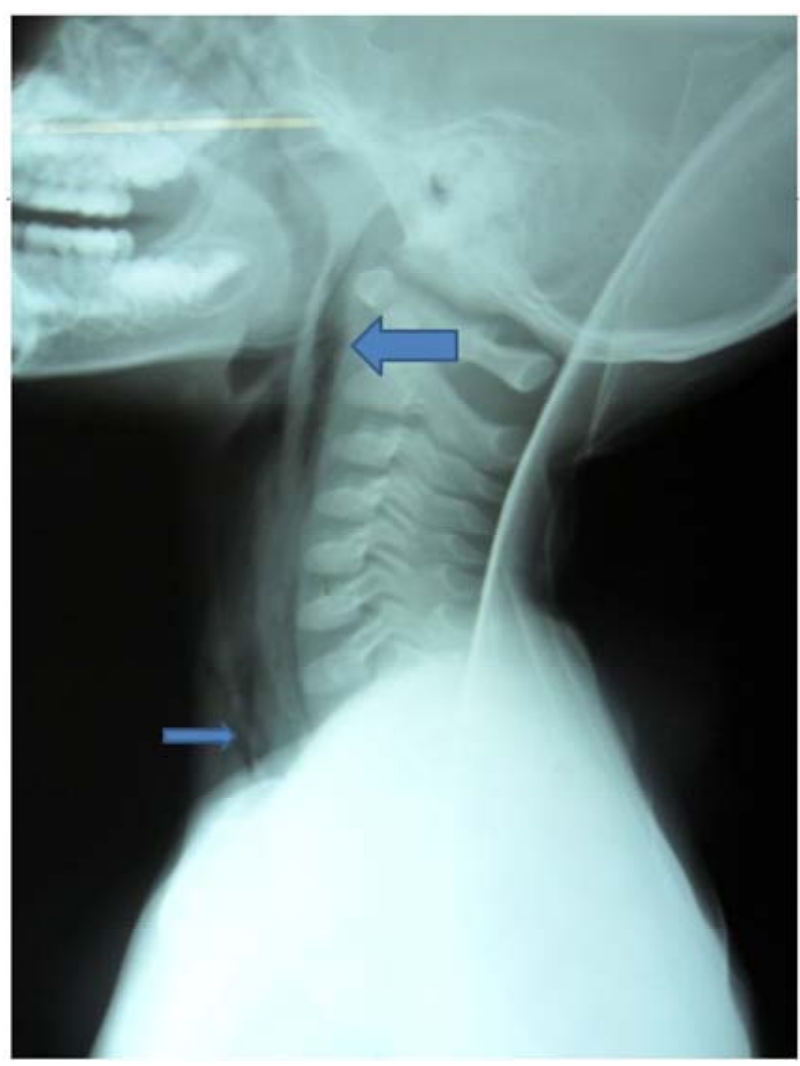

Figure 1. Lateral soft tissue neck plain radiograph showing air in the retropharyngeal space (big arrow) and in the subcutaneous tissue (small arrow).

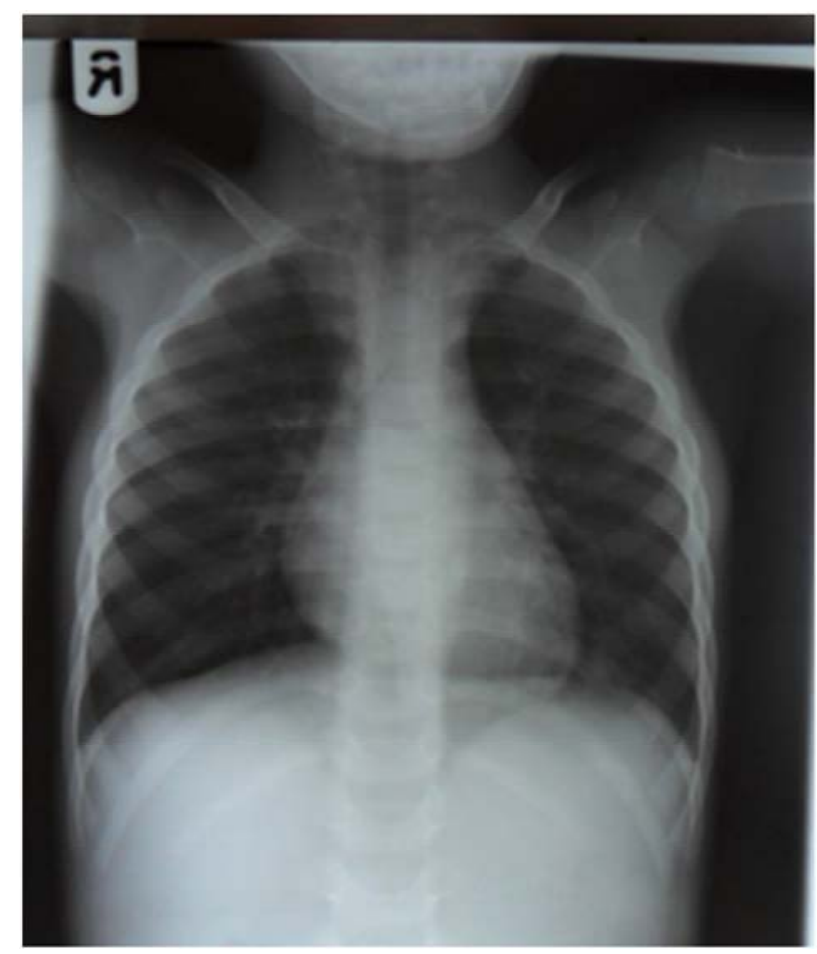

Figure 2. Plain radiograph of the chest showing no evidence of pneumomediastinum. 
vital signs monitoring was instituted. However, preparations for emergency airway intervention via an emergency tracheostomy was considered if his clinical condition had worsened.

Clinically, ronchi improved on chest auscultation and breaths sounds were normal in all the lung fields. There was also gradual improvement in the dyspnoea and no observed increase in the neck swelling within 4 hours of conservative management. Supplemental oxygen was then discontinued when oxygen saturation was $98 \%$ in ambient room air, 36 hours after admission.

A repeat cervical X-ray showed marked reduction in the retropharyngeal emphysema, improvement of the supraglottic region and disappearance of the emphysema of the subcutaneous tissue of the neck (Figure 3).

He was discharged on the third day after admission. His mother was educated on the dangers of this practise. Subsequent follow-up review showed sustained clinical improvement with resolution of the emphysema.

\section{DISCUSSION}

The retropharyngeal space is a potential space located between the middle and deep layers of the deep cervical fascia. It extends from the skull base to the posterior mediastinum at the level of the $2^{\text {nd }}$ thoracic vertebra where the alar fascia and visceral division fuse, where emphysema could result when air dissects this fascial plane.

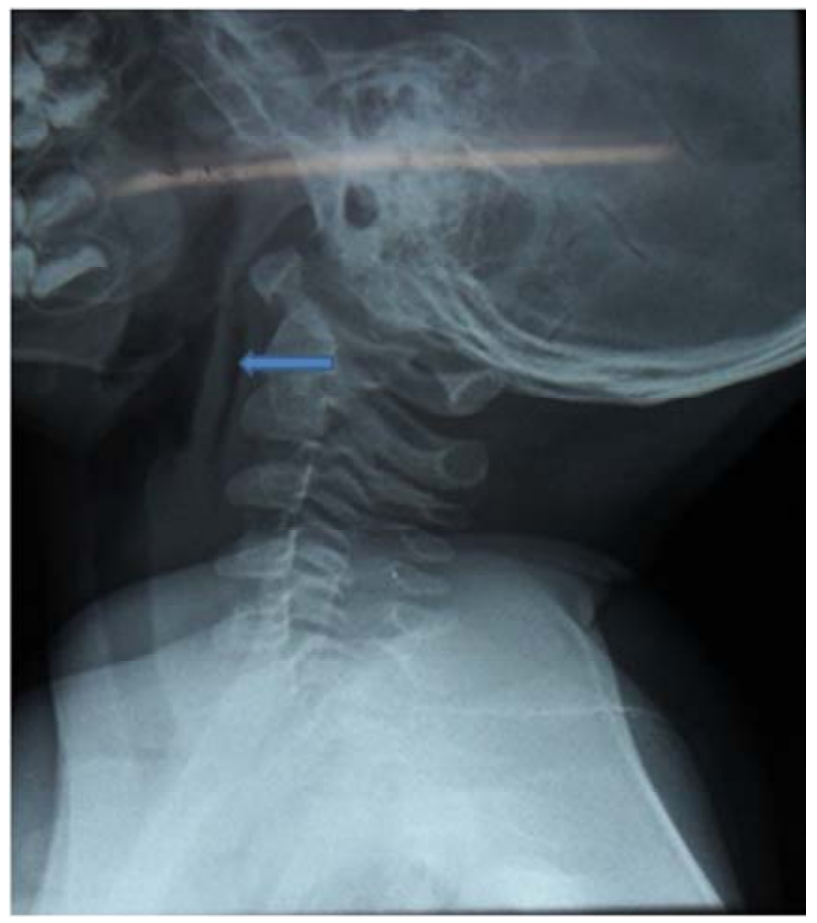

Figure 3. Lateral soft tissue neck plain X-ray showing marked reduction of retropharyngeal Emphysema after 48 hours on conservative treatment (arrow).
Retropharyngeal emphysema is a rare clinical entity. It is rarer still to have the emphysema restricted to this space alone as it is more common for emphysema in the retropharyngeal space to be associated with pneumomedistinum and cervical emphysema. One of the earlier reports of this condition was by Brown in 1949 as a complication following double contrast enema [3].

Dental procedures especially those involved with the used of compressed air drills have been shown to cause emphysema of the retropharyngeal space [4]. This complication has also been reported in the early post operative period following tonsillectomy [5]. These patients typically presented with throat pain, dysphagia and odynophagia. Diagnosis was made by plain radiographs of the neck [4].

Retropharyngeal emphysema has also been described in patients with severe maxillofacial injuries [6]. Traumatic injury to the pharynx or oesophagus in the newborn from intubation or tube suctioning has been documented to cause retropharyngeal emphysema [7]. This may present in various ways. A notable one is difficulty with passing a gastric tube which resulted in a misdiagnosis of oesophageal atresia [7]. More commonly, these patients present with respiratory distress and diagnosis is usually established radiologically [7].

Substance abuse such as free-basing cocaine and ecstasy are rare causes of retropharyngeal emphysema [8]. Onwudike [9] reported dysphagia as the only complaint in a patient with retropharyngeal emphysema following ecstasy abuse (more commonly chest symptoms of pneumomediastinum have been reported with substance abuse). The patient was admitted for observation and given $100 \%$ oxygen [9].

Spontaneous cases of retropharyngeal emphysema have been reported [10]. There is usually associated pneumomediastinum. The symptoms of spontaneous retropharyngeal emphysema are sore throat, dysphagia and generalised neck pain. Physical findings are crepitance in the neck, chest and fascial areas. Radiologically, there are streaks of air within the cervical region and free air in the retropharyngeal region [10].

In this environment, largely due to ignorance and inadequate health care facilities, mothers as well as other child care givers usually make attempts at removal of foreign bodies from the ear, nose and throat. This has been found to be quite common in the low socio economic groups and individuals with poor education. Majority of foreign bodies in the aforementioned locations have also been found to be commoner in children belonging to this socioeconomic group [11].

For removal of foreign bodies from the throat, the attempt described usually entails immediate bending of the child's head forward and in the same movement thrust- 
ing of the fingers into the oropharynx and down to the hypopharynx with sweeping motions. This is aimed at dislodging the foreign agent and retrieving it.

The consequences of this manoeuvre are usually injury to the oral cavity and oropharynx with occasional laryngeal oedema. Majority of these cases are unreported. This is the first case of retropharyngeal emphysema that has been reported as a consequence of this practice.

Isolated cases of retropharyngeal emphysema without attendant pneumomediastinum can be managed conservatively mainly by observation, supplemental oxygen therapy as well as systemic steroids [8,9]. More severe clinical presentations especially upper airway obstruction is managed with urgent tracheostomy, broad-spectrum prophylactic antibiotic coverage and close observation [12]. The airway of children is quite narrow and compromise can have disastrous consequences. In this patient, resolution of this condition was achieved by conservative management however preparations for emergency airway intervention were made and an emergency tracheostomy would have been performed if his clinical condition had worsened.

As was stated in the case report, no foreign body was found and in the follow up visit 2 days after discharge, the patient's mother confessed to having located the bead in the pocket of the pair of shorts the patient had been wearing prior to the incident.

\section{CONCLUSION}

This rare clinical entity is seldom thought of as a differential in the management of patients presenting with acute upper aerodigestive tract conditions. The role of radiology in making a diagnosis cannot be over emphasised. Health education to increase public awareness of the ills of this "socio-cultural" practice is of paramount importance in order to reduce the incidence of this potentially life threatening condition.

\section{CONSENT}

Written informed consent was obtained from the patient for the publication of this case report and accompanying images. A copy of this consent is available for review by the Editor-in-Chief of this journal.

\section{REFERENCES}

[1] Mauder, R.J., Pierson, D.J. and Hudson, L.D. (1984) Subcutaneous and mediastinal emphysema; Pathophysiology, diagnosis and management. Archives of Internal Medicine, 144, 1447-1453. doi:10.1001/archinte.1984.00350190143024

[2] Bhatia, P.L. (1989) Hypopharyngeal and oesophageal foreign bodies. East African Medical Journal, 66, 804-811.

[3] Brown, S. (1949) Diffuse emphysema following a double contrast enema. Radiology, 37, 228-229.

[4] Sausin, L., LaBruna, A., Levine, J. and Komser, A. (1997) Subcutaneous and retropharyngeal emphysema after dental procedures. Otolaryngology, Head and Neck Surgery, 117, 122-123. doi:10.1016/S0194-5998(97)70219-5

[5] Fortes, F., Sennes, L.U., Fortes, F.S.G., Immamura, R. and Tsuyi, D.H. (2007) Cervical emphysema as an early complication of tonsillectomy. International Archives of Otorhinolaryngology, 11, 65-69.

[6] Azenha, M.R., Yamaji, M.A., Avelar, R.L., de Freitas, Q.E., Laureano Filho, J.R. and de Oliveira Neto, P.J. (2011) Retropharyngeal and cervicofacial subcutaneous emphysema after maxillofacial trauma. Oral and Maxillofacial Surgery, 15, 245-249. doi:10.1007/s10006-010-0246-5

[7] Barlev, D.M., Nagourney, B.A. and Saintoge, R. (2003) Traumatic retropharyngeal emphysema as a cause of severe respiratory distress in a new born. Paediatric Radiology, 33, 429-432.

[8] Riccio, J.C. and Abbott, J. (1990) A simple sore throat? Retropharyngeal emphysema secondary to free-basing cocaine. Journal of Emergency Medicine, 8, 709-712. doi:10.1016/0736-4679(90)90283-2

[9] Onwudike, M. (1996) Ecstasy induced retropharyngeal emphysema. Journal of Accident and Emergency Medicine, 13, 359-361. doi:10.1136/emj.13.5.359

[10] Wu, Z.B., Patel, N. and Holliday, R. (2006) Spontaneous Retropharyngeal emphysema. Otolaryngology, Head and Neck Surgery, 134, 170-172. doi:10.1016/j.otohns.2004.12.006

[11] Mukherjee, A., Haldar, D., Dutta, S., Dutta, M., Saha, J. and Sinha, R. (2011) Ear, nose and throat foreign bodies in children: A search for socio-demographic correlates. International Journal of Paediatric Otorhinolaryngology, 75, 510-512. doi:10.1016/j.ijporl.2011.01.006

[12] Breznick, D.A. and Saporito, J.L. (1989) Iatrogenic retropharyngeal emphysema with impending airway obstruction. Otolaryngology, Head and Neck Surgery, 115, 13671372. doi:10.1001/archotol.1989.01860350101024 